Check for updates

Cite this: RSC Adv., 2018, 8, 4548

Received 30th October 2017

Accepted 6th January 2018

DOI: 10.1039/c7ra11954b

rsc.li/rsc-advances

\section{Promoting early neovascularization of SIS-repaired abdominal wall by controlled release of bioactive VEGF $\uparrow$}

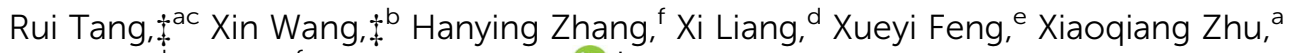 \\ Xinwu Lu, ${ }^{b}$ Fei $W^{f}$ and Zhengni Liu (iD *a
}

Insufficient early neovascularization post-operation is thought to be the main reason of surgical recurrence of porcine small intestinal submucosa (SIS)-repaired abdominal wall defects. The controlled release of exogenous angiogenic growth factors (GFs) from biocompatible carriers is a possible way to solve this problem. In the present study, dextran nanoparticles (DNPs) loaded with vascular endothelial growth factor $165\left(\mathrm{VEGF}_{165}\right)$ were pre-formulated by dual-aqueous phase separation method and then electrospun into the poly(lactic-co-glycolic acid) (PLGA) polymer fibers. The aim of this material is to release VEGF in a sustained manner with the degradation of PLGA and maintain its bioactivity concurrently. The prepared VEGF/DNPs-PLGA membrane was sandwiched by dual-layer SIS to construct a SIS-DNPS/VEGF-PLGA-SIS (SVDPS) composite scaffold. The in vitro study showed that the VEGF/ DNPs-PLGA obtained higher VEGF encapsulation efficiency as well as better release property and bioactivity than the emulsion electrospun VEGF-PLGA and PLGA fibrous membranes by ELISA and HUVEC proliferation. The in vivo study showed that the SVDPS composite scaffold promoted significantly higher early therapeutic neovascularization within 2 weeks post-surgery than SIS-VEGF-PLGA-SIS (SVPS) and SIS-PLGA-SIS (SPS) by immunohistochemical and immunoblotting examination.

\section{Introduction}

The application of meshes for repairing abdominal wall defects caused by trauma, extensive surgical tumor resection, or hernias has been widely accepted by surgeons. To date, both synthetic and biological meshes (biomesh) are commonly

${ }^{a}$ Department of Hernia and Abdominal Wall Surgery, Shanghai East Hospital, Tongji University, 150 Ji Mo Road, Shanghai 200120, PR China. E-mail: kevintown1972@ 126.com; zhuxq77@163.com; liuzhengni@gmail.com; Fax: +86 21 61569272; Tel: +862161569272

${ }^{b}$ Department of Vascular Surgery, Shanghai Ninth People's Hospital, Shanghai Jiao Tong University School of Medicine, 639 Zhi Zao Ju Road, Shanghai 200001, PR China.E-mail:wangxin2012@sjtu.edu.cn; luxinwu@shsmu.edu.cn

'Department of General Surgery, Shanghai Ninth People's Hospital, Shanghai Jiao Tong University School of Medicine, 639 Zhi Zao Ju Road, Shanghai 200001, PR China ${ }^{d}$ Department of Thoracic Surgery, Shanghai Ninth People's Hospital, Shanghai Jiao Tong University School of Medicine, 639 Zhi Zao Ju Road, Shanghai 200001, PR China.E-mail: youyouxiaoqu@163.com

${ }^{e}$ Department of General Surgery, Lu'an People's Hospital, Lu'an Affiliated Hospital of Anhui Medical University, 21 West Wan Xi road, Lu'an, Anhui 237000, PR China. E-mail: fxy514270277@139.com

${ }^{f}$ School of Pharmacy, Shanghai Jiao Tong University, 800 Dongchuan Road, Shanghai 200240, PR China. E-mail: zhhany@sjtu.edu.cn; feiwu@sjtu.edu.cn; Fax: +86 21 34205072; Tel: +862134204695

$\dagger$ Electronic supplementary information (ESI) available. See DOI: 10.1039/c7ra11954b

\$ These authors contributed equally to this work. available in the clinic. Biomeshes are biodegradable and biocompatible extracellular matrices (ECM) that possess the scaffold structure from animal sources. They possess the potential for long-lasting repair of the abdominal wall defect with fewer complications, while synthetics tend to cause infection, visceral adhesion and erosion, ${ }^{\mathbf{1 - 4}}$ and even mesh-related cancer. ${ }^{5}$ Among these biological scaffolds, porcine small intestinal submucosa (SIS) has a three-dimensional (3D) structure to facilitate cell seeding, migration, proliferation, and differentiation. $^{6-9}$ The scaffold is finally replaced by new site-specific tissues without any residuum, which is defined as tissue endogenous regeneration. ${ }^{\mathbf{1 0 , 1 1}}$ The strength of the reconstructed tissue depends on the balance between the rate of biological scaffolds degradation and that of new tissue generated and incorporated, replacing degraded scaffolds, also defined as remodeling..$^{12}$ If the remodeling process does not outpace the degradation, later repair failure is inevitable. The neovascularization of SIS is better than that of other sources of biological scaffolds such as the pericardium and acellular dermal matrix ${ }^{\mathbf{1 3 - 1 5}}$ which is a vital biological process in the whole regeneration and remodeling process. However, the short- or long-term bulge, herniation, or recurrence rate after SIS repair suggest that the neovascularization is still insufficient for tissue regeneration. ${ }^{\mathbf{1 6}-18}$ Therefore, improving the scaffoldinduced endogenous regeneration through rapid angiogenesis 
within tissue-engineered constructs remains indispensable to improve its clinical efficacy.

To promote early neovascularization of SIS, delivery and controlled release of bioactive exogenous angiogenic GFs such as vascular endothelial growth factor $165\left(\mathrm{VEGF}_{165}\right)$ with a nontoxic and degradable carrier is a practicable method. Poly(lacticco-glycolic) acid (PLGA) and other polymer materials are frequently used to carry drugs or proteins in such a controlled release system. ${ }^{19-21}$ However, to establish a successful PLGAbased release system, GFs must be preserved from hazardous conditions to avoid loss of bioactivity. It has been testified that loading bare GFs into hydrophobic polymeric matrix directly leads to a huge protein waste of up to $40 \%{ }^{22}$ Furthermore, the delicate advanced structure of GFs is susceptible to the organic phase and to water/oil or water/air interfacial tension, ${ }^{23-25}$ which are known factors leading to protein denaturation.

Aqueous-aqueous freezing-induced phase separation (shorted as "aqueous method" hereafter) ${ }^{25,26}$ has been used in our previous study to prepare polysaccharide micro-particles or nano-particles containing structurally delicate agents. GFs can be encapsulated into the dextran nanoparticles (DNPs) and formed GFs/DNPs without any contact with the water/oil interface, organic solvents, or polymers by this aqueous method. The pre-formulated GFs/DNPs can further be loaded into PLGA by various techniques to form the structures or shapes of interest. The aqueous method was used to release the proteins such as granulocyte-macrophage colony-stimulating factor (GM-CSF), granulocyte colony-stimulating factor (G-CSF), $\beta$-galactosidase, myoglobin, bovine serum albumin (BSA) successfully, ${ }^{25}$ and basic fibroblast GF (bFGF) ${ }^{27}$ from polymer carriers can be controlled and their bioactivity is maintained.

In this study, to promote the early neovascularization of SISrepaired abdominal wall with controlled release of bioactive VEGF, we first embedded VEGF into DNPs to form VEGF/DNPs via the aqueous method. The pre-formed VEGF/DNPs were fabricated into PLGA fibers (functioning as the VEGF carrier) via co-electrospinning to fabricated a VEGF/DNPs/PLGA membrane. Subsequently, the VEGF/DNPs/PLGA membrane was sandwiched between two layers of SIS to construct a composite scaffold and applied to repair abdominal wall defect of a Sprague-Dawley (SD) rat model. The in vitro properties of the VEGF/DNPS/PLGA carrier, the bioactivity of VEGF released from VEGF/DNPs/PLGA, and the in vivo effects of the composite scaffold after implantation were evaluated. We hypothesized that VEGF could be released from VEGF/DNPs/ PLGA or the composite mesh in a sustained manner while retaining its bioactivity. Moreover, early stage improved neovascularization was achieved, followed by enhanced collagen deposition and mechanical properties.

\section{Materials and methods}

\subsection{Materials}

PLGA (monomer ratio: 50/50, $M_{\mathrm{w}}: 40000$ ) was obtained from Lakeshore Biomaterials, Inc. (Birmingham, AL, USA). Dextran ( $\left.M_{\mathrm{w}}: 6000-76000\right)$ and polyethylene glycol (PEG) $\left(M_{\mathrm{w}}: 6000\right)$ were from Sigma Chemical Co, (St Louis, MO, USA). $N, N-$
Dimethyl formamide (DMF) and tetrahydrofuran (THF) of analytical grade were obtained from Sinopharm Chemical Reagent Co., Ltd, (Shanghai, China). Recombinant human VEGF $_{165}$ and VEGF enzyme-linked immunosorbent assay (ELISA) kits were purchased from R\&D Systems, Inc. (Minneapolis, MN, USA) and used according to the manufacturer's instructions. Cell Counting Kit-8 assay kit (CCK-8) was from Dojindo Molecular Technologies, Inc. (Kumamoto, Japan). A human umbilical vein endothelial cell (HUVEC) line and EBM-2 medium were procured from the American Type Culture Collection (Manassas, VA, USA). Mouse monoclonal anti-CD31 and rabbit polyclonal anti-fibroblast-specific protein 1 (FSP1) were purchased from Abcam, Inc. (Cambridge, MA, USA). Mouse polyclonal anti-CD68, BCA protein assay kit, and $\beta$-actin antibody were obtained from Santa Cruz Biotechnology, Inc. (Dallas, TX, USA). Goat anti-mouse and goat anti-rabbit IgG horseradish peroxidase-linked secondary antibody was from Boster Biosciences (Wuhan, China). Other reagents such as $0.5 \%$ sodium dodecyl sulfate (SDS) and $0.2 \%$ Triton X-100 were provided by the Tissue Engineering Laboratory of Shanghai Jiaotong University School of Medicine.

\subsection{Preparation and characterization of SIS}

Fresh jejunum was obtained from healthy 6 month-old pigs (weight $100-120 \mathrm{~kg}$ ) and prepared as previously described. ${ }^{14}$ In brief, the mucosa, serosa, and tunica muscularis were mechanically removed. The submucosa layer was chemically treated with SDS and Triton X-100 sequentially for decellularization and then freeze-dried and sterilized with $\gamma$-rays (25 kGy; $60 \mathrm{Co}$ ). For histological examination, monolayer SIS was stained with hematoxylin and eosin (H\&E).

\subsection{Preparation of VEGF/DNPs}

VEGF was encapsulated into dextran nanoparticles using the aqueous method. The procedure was performed as previously described. $^{25,26,28}$ VEGF was first dissolved into dextran aqueous solution $(5 \%(\mathrm{w} / \mathrm{w}))$, and then added into PEG aqueous solution $(5 \%(\mathrm{w} / \mathrm{w}))$ (mass ratio, VEGF: dextran: PEG $=1: 4000: 40$ 000). The two liquors were mixed by vortexing to form a clear solution and the mixture was frozen at $-80{ }^{\circ} \mathrm{C}$ for $12 \mathrm{~h}$. The frozen solution was then lyophilized for $24 \mathrm{~h}$. At last, the dried powder was washed with dichloromethane, and centrifuged (12 $000 \mathrm{rpm}, 5 \mathrm{~min}$, repeated 3 times) to remove PEG. The VEGF/DNPs were collected and stored at $20^{\circ} \mathrm{C}$.

2.3.1 Encapsulation efficiency of VEGF and recovery efficiency of DNPs. VEGF/DNPs were dissolved in phosphate buffer saline (PBS) and the actual amount of encapsulated VEGF was determined using an ELISA kit. Encapsulation efficiency is the percentage of the genuine protein to theoretical protein, i.e., encapsulation efficiency $=$ (genuine protein recovered from VEGF/DNPs/theoretical protein) $\times 100 \%$. The recovery efficiency is the percentage of the genuine weight of collected VEGF/DNPs to the theoretical weight of VEGF/DNPs, i.e., recovery efficiency $=$ (genuine VEGF/DNPs/theoretical VEGF/ DNPs) $\times 100 \%$. 
2.3.2 Scanning electron microscopy (SEM) for VEGF/DNPs. VEGF/DNPs were characterized by using SEM (FEISIRION 2000 SEM system, Beaverton, OR, USA). Briefly, the samples were fixed on a metal stub with electric conduction paste and sputtercoated with gold. The morphology and size of the nanoparticles were observed and images were taken.

\subsection{Electrospinning of PLGA fibrous membranes}

Three groups of PLGA membranes were fabricated: (a) for VEGF/DNPs-loaded PLGA membrane (VEGF/DNPs-PLGA), $500 \mathrm{mg}$ PLGA was dissolved using $2 \mathrm{~mL}$ of the mixed organic solution of DMF and THF ( $/ v=1: 3)$, then magnetically stirred until fully dissolved. The theoretical weight ratio of VEGF and PLGA was set to $1: 10$ 000; the amount of VEGF/DNPs needed in this process was calculated according to the ratio of VEGF versus dextran mentioned above. The VEGF/DNPs were dispersed into PLGA solution, magnetically stirred $(1000 \mathrm{rpm}$, $30 \mathrm{~min}$ ), and then loaded into a $2 \mathrm{~mL}$ syringe. The syringe was subsequently placed before the pump of an electrostatic spinning machine (Yongkang Leye Science and Technology Development Co., Ltd., Beijing, China). The distance between the collection plate and the needle tip, the voltage, and the feeding rate was set as $15 \mathrm{~cm}, 10 \mathrm{kV}$, and $1 \mathrm{~mL} \mathrm{~h}^{-1}$, respectively. (b) For VEGF-loaded PLGA membrane (VEGF-PLGA), VEGF was first dissolved in PBS to obtain a $0.5 \mu \mathrm{g} \mu \mathrm{L}^{-1}$ suspension, gradually dropped into PLGA solution, and electrospun under the same conditions of group a. (c) For blank PLGA membrane, the PLGA solution without VEGF and dextran was directly electrospun.

2.4.1 Encapsulation efficiency of PLGA membranes. To determine the encapsulation efficiency, VEGF/DNPs-LGA and VEGF-PLGA were respectively dissolved by DCM in a $2 \mathrm{~mL}$ centrifuge tube, followed by centrifugation at $12000 \mathrm{rpm}$ for 5 min and repeated 3 times. The supernatant was aspirated and the centrifuge tube was placed in a fume cupboard until DCM evaporated. The residual sediment was dissolved with PBS. An ELISA kit was used to detect VEGF content and the encapsulation efficiency was calculated.

2.4.2 Morphology and structure of PLGA membranes. The morphology and structure of PLGA membranes were determined by SEM. PLGA samples were placed on a metal stub, fixed with electric conduction paste, and sputter-coated with gold. The substructure of different samples was observed and the images were recorded. Furthermore, the water contact angle was determined using a Kruss GmbH DSA 100 Mk 2 goniometer (Hamburg, Germany).

\subsection{Construction of sandwiched composite scaffold}

PLGA-contained membranes were inserted into the two layers of SIS $\left(4 \times 3 \mathrm{~cm}^{2}\right)$ to construct different sandwiched composite scaffolds. SIS-VEGF/DNPs-PLGA-SIS (SVDPS), SISVEGF/PLGA-SIS (SVPS), and SIS-PLGA-SIS (SPS) were prepared. To avoid any other exposure factors, no suture, adhesive agent, or other mechanical measure was used for scaffold fixation.

\subsection{In vitro VEGF release from PLGA membranes}

To assess the in vitro release kinetics of VEGF from different groups, VEGF/DNPs-PLGA and VEGF-PLGA membranes of equal weight $(10 \mathrm{mg})$ were placed in $10 \mathrm{~mL}$ of PBS at $37{ }^{\circ} \mathrm{C}$ and agitated continuously at $150 \mathrm{rpm}$. The supernatant was collected daily and fresh PBS of equal volume was added until the $20^{\text {th }}$ day. To determine whether SIS would affect the controlled release of VEGF/DNPs-PLGA and VEGF-PLGA membranes, we also assessed the release kinetics of SVDPS and SVPS membranes. The amount of VEGF released from different membranes was measured using the VEGF ELISA kit and the release rate was calculated. The theoretical release ratio is the percentage of the genuine weight of collected VEGF to the theoretical weight of VEGF encapsulated in $10 \mathrm{mg}$ VEGF/DNPsPLGA or VEGF-PLGA membranes.

\subsection{In vitro cell proliferation and VEGF bioactivity assay}

Sterilized SVDPS, VEGF/DNPs-PLGA, SVPS, VEGF-PLGA, PLGA, and SIS were respectively cultured with $3 \mathrm{~mL}$ EBM-2 serum- and cytokine-free medium at $4{ }^{\circ} \mathrm{C}$, and agitated 3 days at $150 \mathrm{rpm}$. The daily release solutions from separate groups were extracted for two weeks to assess their effects on the proliferation of HUVECs. The endothelial cells (ECs) were suspended in the release solutions with $0.5 \% \mathrm{FBS}$ and seeded in a 96-well plate (20000 cells per $\mathrm{mL}$ ) and then incubated in $\mathrm{CO}_{2} /$ air $(5: 95, \mathrm{v} / \mathrm{v})$ at $37^{\circ} \mathrm{C}$ for $48 \mathrm{~h}$ without medium change. The proliferation efficiency of HUVECs was tested using CCK-8 assay. A microplate reader (Thermo Fisher scientific, Waltham, MA USA) was used to assess the optical density (OD) at a wavelength of $450 \mathrm{~nm}$.

The bioactivity of the VEGF recovered during the process was also determined by assessing the proliferation effect on HUVECs using CCK8 assay. The standard protein solution was used in the bioactivity assay as a positive control.

\subsection{In vivo evaluation of the composite scaffolds in an animal model}

This study was performed in strict accordance with the National Institutes of Health's Guide for the Care and Use of Laboratory Animals (NIH Publication N01-OD-4-2139, Rev. 2011) and was approved by the Institutional review committee of Shanghai Jiao Tong University School of Medicine (ID: SYXK 2008-0050, Shanghai, China). The entire processes were approved and carried out by Shanghai Key Laboratory of Tissue Engineering (Shanghai, China). One hundred and twenty male SpragueDawley rats $(250 \pm 30 \mathrm{~g})$ (SLAC National Rodent Laboratory Animal Resources, Shanghai, China) were randomly divided into four groups: SVDPS, SVPS, SPS, and SIS. An abdominal wall defect was created and repaired instantly with the materials previously prepared. Rats were euthanized at 1, 2, 4, 8, and 12 weeks post-surgery for gross observation, histological examination, and biomechanical tests.

2.8.1 Surgical procedure. Animals were anesthetized by intraperitoneal injection of pentobarbital sodium (40 $\left.\mathrm{mg} \mathrm{kg}^{-1}\right)$ and then immobilized on the operating table, with the 
abdomen shaved and disinfected with $0.5 \%$ povidone-iodine. A $3 \times 2 \mathrm{~cm}^{2}$ full-defect except the skin was created. Meshes were hydrated in sterile saline for $1 \mathrm{~min}$ before use. They were fixed using intraperitoneal underlay technique and sutured intermittently with 3-0 polypropylene. The skin was interruptedly closed with 4-0 polypropylene suture.

2.8.2 Macroscopic observation. After operation, any signs of wound infection, dehiscence, hematoma, seroma, bulge, or herniation were recorded. At each sampling time, six animals in each group were randomly euthanized by overdose of pentobarbital sodium. Visceral adhesion, including the area and tenacity, was also evaluated according to the following criteria: 0 , no adhesion; 1 , surface area $<25 \%$, freed by blunt dissection; 2 , surface area $<50 \%$, freed by aggressive dissection; 3 , surface area $<75 \%$, freed by sharp dissection; and 4 , surface area $\geq 75 \%{ }^{34}$ Ten random measurements of thickness over the central area of the explant and its mean were noted in order to calculate the proportional change in thickness. It was defined as: the mean value measured at the time of sacrifice - the original thickness of the implant. The implant dimensions were measured using a centimeter scale and recorded to assess any subsequent intra-corporal contraction. The percentage of contraction were recorded as the changes in dimensions/ original dimensions.

2.8.3 Histological and immunohistochemical examination. At euthanasia, the scaffold $(40 \mathrm{~mm})$ plus $5 \mathrm{~mm}$ of the surrounding tissues at both ends were collected as the samples $\left(50 \times 10 \mathrm{~mm}^{2}\right)$. Samples were fixed with $4 \%$ paraformaldehyde for $24 \mathrm{~h}$, dehydrated and embedded in paraffin, and sectioned into $5 \mu \mathrm{m}$-thick slices. H\&E staining was used to observe the general morphology of the repaired site, including cell infiltration, tissue incorporation, angiogenesis, and inflammation. Qualitative assessment of collagen deposition was performed using Masson trichrome staining. The total collagen content was reported as a percentage of the aniline green staining divided by the total tissue area of the section using Image $\mathrm{J}$ software (National Institutes of Health, Bethesda, MD, USA). ${ }^{29}$

Immunohistochemical staining was performed using BondMax system (Leica, Wetzlar, Germany). Blood vessel density was assessed by anti-CD31 antibody staining. Fibroblasts were visualized by anti-FSP1 antibody staining. ${ }^{30}$ The host immune response represented by macrophage infiltration was evaluated by anti-CD68 antibody staining. Quantification of the blood vessels, fibroblasts, and macrophages was performed by counting positively stained cells at $400 \times$ magnification. In each type of staining, six randomized chosen fields in each section were analyzed by two blinded investigators by using Image-Pro Plus software 6.0 (Media Cybernetics, Inc., Silver Spring, MD, USA).

2.8.4 Western blotting analysis. For immunoblotting analysis, proteins were extracted from the homogenates after incubation in lysis buffer containing protease inhibitors and detergents, following centrifugation at $12000 \mathrm{rpm}$ for $10 \mathrm{~min}$. Protein concentrations were determined by using the BCA protein assay kit. Equal amounts of protein were electrophoresed by $10 \%$ SDS-PAGE and transferred to PVDF membranes. The proteins were probed with CD31 and $\beta$-actin antibodies, followed by incubation with the peroxidase-conjugated secondary antibody at $37^{\circ} \mathrm{C}$ for $1 \mathrm{~h}$. The membrane was washed three times and the protein bands were visualized. CD31 level was quantified and normalized to $\beta$-actin bands by densitometry in Photoshop 8.0 (Adobe, San Jose, CA, USA).

2.8.5 Examination of mechanical properties. The in vitro scaffolds ( $50 \times 10 \mathrm{~mm}^{2}, n=6$ in each group) or in vivo explants $\left(50 \times 10 \mathrm{~mm}^{2}, n=4-6\right.$ in each group) were subjected to mechanical tests using a biomechanical analyzer (Instron 4411, Canton, MA, USA) and the length of tested samples was set at $10 \mathrm{~mm}$ between two grippers as previously reported. ${ }^{31,32}$ The grippers were gradually moved apart at a speed of $10 \mathrm{~mm} \mathrm{~min}^{-1}$ until complete rupture of tested tissue to generate the data of maximal load (max load in Newton [N]). Young's modulus (MPa) was calculated from the linear slope based on the created stress-strain curves and the tensile strength was generated from the maximal load divided by the cross-sectional area (MPa). The stiffness (Newton per millimeter) was determined by calculating the slope of the load (linear portion) vs. displacement plot. All data were presented as mean \pm standard deviation.

\subsection{Statistical analysis}

Measurement data were recorded as mean \pm standard deviation and analyzed by two-tailed student's $t$-test or one-way analysis of variance followed by the Student-Newman-Keuls (SNK) post hoc test. Ranked data were analyzed by using Kruskal-Wallis test. SPSS version 19.0 software (SPSS Inc., Chicago, IL, USA) was used for statistical analysis. $P<0.05$ was considered statistically significant.

\section{Results}

\subsection{Characteristics of SIS}

A monolayer of SIS was successfully prepared (Fig. 1A). H\&E staining revealed no remaining cellular nucleus inside (Fig. 1B). Dual-layer SIS was constructed and cut into pieces of $4 \times 3 \mathrm{~cm}^{2}$. The max load, Young's modulus, tensile strength, stiffness of the dual-layer SIS in vitro were $4.89 \pm 0.91 \mathrm{~N}, 3.82 \pm 1.30 \mathrm{MPa}$, $6.01 \pm 2.71 \mathrm{MPa}, 4.88 \pm 1.01 \mathrm{~N} \mathrm{~mm}^{-1}$, respectively (Table 1).

\subsection{Characteristics of VEGF/DNPs}

VEGF/DNPs were successfully prepared by using the freezinginduced phase separation method. The recovery and encapsulation efficiencies were $90.42 \pm 8.76 \%$ and $65.94 \pm 9.93 \%$, respectively $(p<0.01)$, which were similar to our previous data. ${ }^{27}$ The results indicated that the aqueous method embedded VEGF efficiently. SEM (Fig. 1C) showed that the nanoparticles presented a smooth surface and the size ranged from 200-1200 nm with an average size of about $503.52 \pm 292.64 \mathrm{~nm}$.

\subsection{Characterization of the electrospun PLGA membranes}

VEGF/DNPs-PLGA, VEGF-PLGA, and pure PLGA fibrous membranes were successfully fabricated. The thickness was $0.11 \pm 0.02 \mathrm{~mm}$. The substructure of PLGA membrane was determined by using SEM (Fig. 1D-F). The protein encapsulation efficiency of the VEGF/DNPs-PLGA membrane reached $37.64 \pm 13.45 \%$, while that of the VEGF-PLGA membrane was 


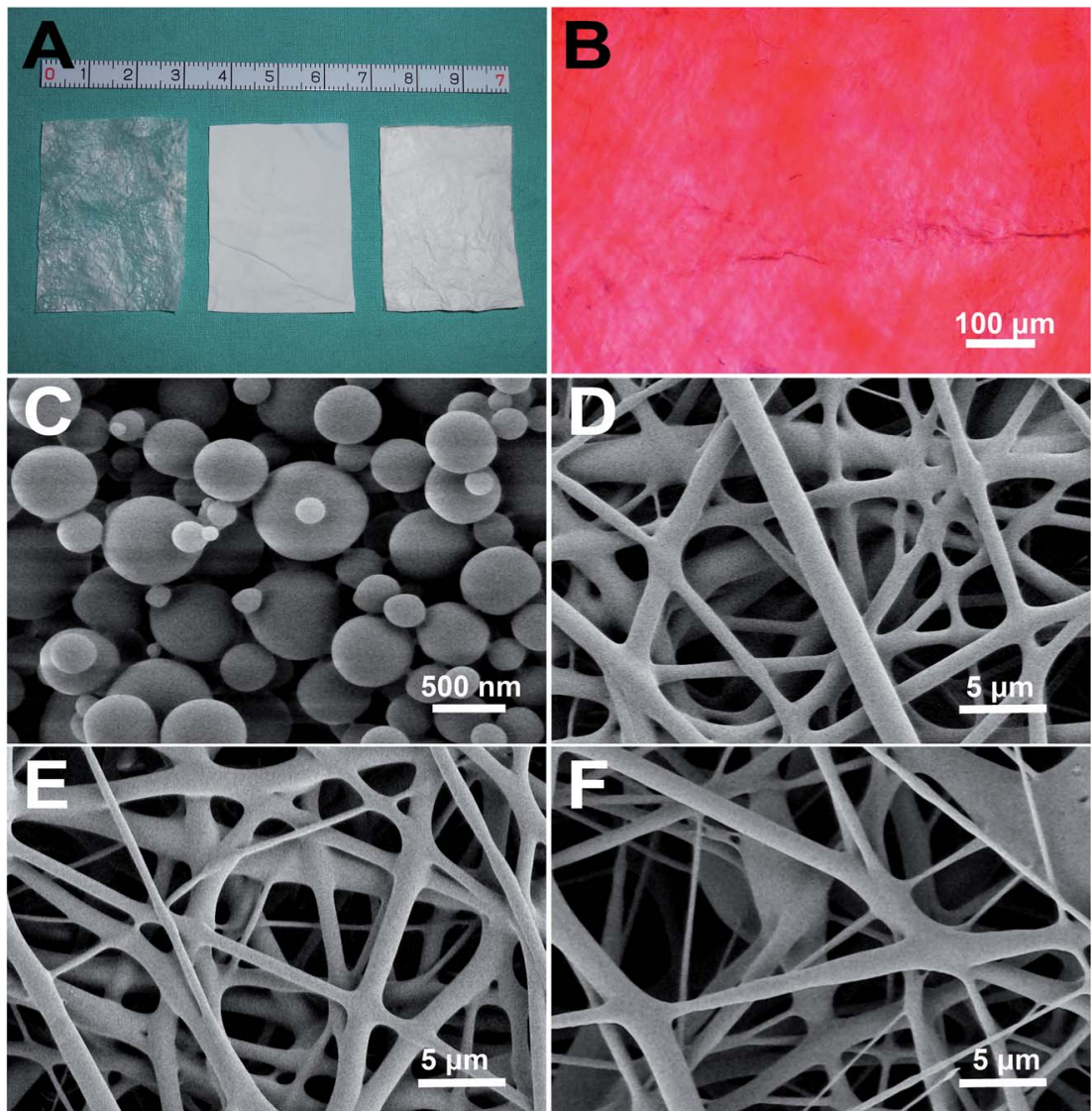

Fig. 1 Macro and micro appearances of various membranes. (A, left) SIS is thin and translucent with an irregular texture; (A, middle) electrospun PLGA fibrous membranes with various contents show similar exterior appearances as in this figure, they were smooth and pure white; (A, right) the sandwich composite mesh constructed by a PLGA membrane and dual-SIS shows no visual difference. (B) H\&E staining of SIS shows no remaining cell nucleus. Magnification: $100 \times$, scale bar $=100 \mu \mathrm{m}$. (C) SEM images of VEGF/DNPs. Magnification: $10000 \times$, scale bar $=500 \mathrm{~nm}$. SEM images of PLGA membrane (D), VEGF-PLGA membrane (E), and VEGF/DNPs-PLGA membrane (F). Magnification (D-F): 3000×, scale bar = $5 \mu \mathrm{m}$.

Table 1 Mechanical properties of SIS, SPS, SVPS and SVDPS in vitro

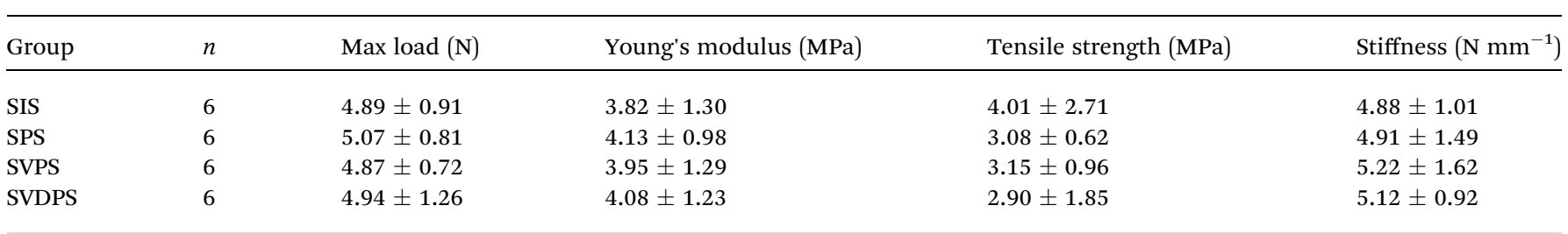

$25.64 \pm 6.02 \%(p<0.05)$. The encapsulation efficiency of the VEGF/DNPs-PLGA membrane was much higher than that of the VEGF-PLGA membrane, suggesting that VEGF was well protected by dextran. The water contact angles of VEGF/DNPsPLGA, VEGF-PLGA, and blank PLGA membranes were $119.7 \pm$ $5.43^{\circ}, 136.1 \pm 8.68^{\circ}$, and $140.0 \pm 8.65^{\circ}$, respectively $(p<0.01)$, indicating that the hydrophilic property of PLGA membrane was also improved after dextran was embedded inside.

\subsection{Macroscopy and mechanical properties of the sandwich composite scaffold}

The sandwich composite scaffolds, SVDPS, SVPS, and SPS were successfully constructed with consistent extrinsic features
(Fig. 1A, right). All composite scaffolds were tested in vitro for mechanical properties in comparison with SIS. Owing to the inconsistent ductility and tenacity between PLGA and SIS, PLGA broke earlier than SIS. As shown in Table 1, the mean max load, Young's modulus, tensile strength and stiffness of SVDPS, SVPS, SPS and SIS scaffolds showed no significant difference among groups $(p>0.01)$.

\subsection{In vitro $\mathrm{VEGF}$ release}

The in vitro release kinetics of VEGF from SVDPS, VEGF/DNPsPLGA, SVPS, VEGF-PLGA, and SIS are shown in Fig. 2. The cumulative quantity of VEGF released from VEGF/DNPs-PLGA and VEGF-PLGA membranes were $365.49 \pm 25.06 \mathrm{ng}$ and 


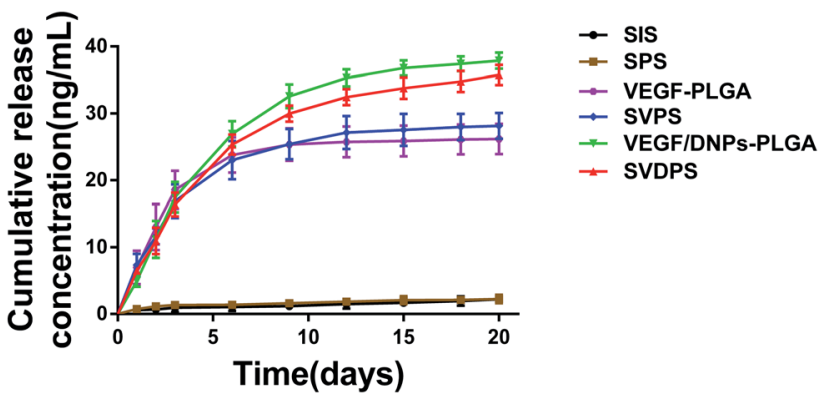

Fig. 2 Cumulative release of VEGF from various PLGA membranes. The release of VEGF/DNPS-PLGA membrane (green inverted triangle) and SVDPS (red triangle) lasted 20 days, while that of VEGF-PLGA (purple hexagon) and SVPS (blue rhombus) membrane only reached 14 days. The VEGF released from the two groups in the first 3 days accounted for $44.39 \pm 4.72 \%$ and $67.25 \pm 13.00 \%$ of the total amount, respectively. VEGF-PLGA showed a more severe burst release phenomenon than the VEGF/DNPs-PLGA membrane. SIS (black round) and SPS (Brown square) were used as controls.

$175.86 \pm 10.56 \mathrm{ng}$, respectively $(p<0.01)$. This demonstrated that $36.55 \pm 2.50 \%$ and $17.59 \pm 1.06 \%$ of the theoretically loaded VEGF was released from the two groups within 21 days. The cumulative quantity of VEGF released from SVDPS and SVPS composite scaffolds were comparable to that of VEGF/ DNPs-PLGA and VEGF-PLGA membranes, which were 357.63 $\pm 15.16 \mathrm{ng}$ and $198.38 \pm 18.43 \mathrm{ng}$, respectively $(p<0.0001)$, indicating that SIS did not significantly affect the controlled release of VEGF from PLGA membranes. The proteins in both PLGA membranes were released in a sustained manner during the test. However, VEGF-PLGA membrane showed a more significant burst release phenomenon that the VEGF/DNPsPLGA membrane in the initial stage. The VEGF released from VEGF/DNPs-PLGA and VEGF-PLGA groups in the first 3 days accounted for $44.39 \pm 4.72 \%$ and $67.25 \pm 13.00 \%$ of the total amount, respectively ( $p<0.01$ ), while it was $43.96 \pm 3.37 \%$ and $59.59 \pm 10.14 \%$ in the SVDPS and SVPS groups, respectively $(p<0.05)$. VEGF was barely detected after 14 days in VEGF-PLGA and SVPS membranes, while VEGF release from VEGF/DNPsPLGA and SVDPS membranes was still detectable at 20 days.

\subsection{In vitro cell proliferation and VEGF bioactivity}

We further studied the effect of the released VEGF on HUVEC proliferation. The released solution from SVDPS and VEGF/ DNPs-PLGA membranes potently induced cell proliferation compared with that from the SIS groups within 14 days. Although the SVPS and VEGF-PLGA release solutions also promoted cell proliferation in 2 weeks, the efficiency decreased after 1 week when compared to that of the released solution from SVDPS and VEGF/DNPs-PLGA groups (Fig. 3A). The VEGF bioactivity results indicated that the proteins recovered from the VEGF/DNPs-PLGA membranes showed a much higher bioactivity than those recovered from VEGF-PLGA (Fig. 3B). These results further confirmed that VEGF bioactivity could be preserved after encapsulation into the dextran nanoparticles during the preparation process.

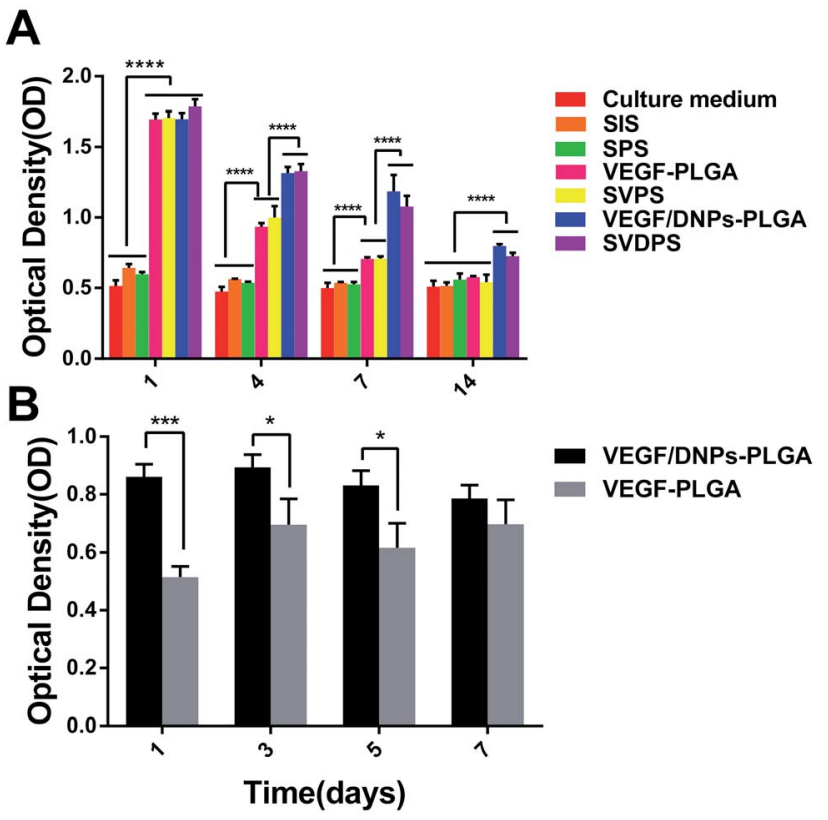

Fig. 3 Determination of in vitro HUVEC proliferation (A) and bioactivity of VEGF recovered from VEGF/DNPs-PLGA and VEGF-PLGA (B). $* * * P<0.0001 ; * * * P<0.001 ; * P<0.05$

\subsection{In vivo evaluation of the composite scaffolds}

3.7.1 Macroscopic observation. To evaluate clinical signs of wound infection, seroma, or dehiscence in each group, animals were observed daily post-surgery for the first 14 days and twice a week afterwards. In the first week, seroma appeared in 5, 3, 4, and 4 animals in the SVDPS, SVPS, SPS, and SIS group, respectively $(p>0.05)$. No seroma was observed at 2 weeks postsurgery. One rat in the SVDPS group died on the $6^{\text {th }}$ day postsurgery because of severe bleeding. It could be caused by the failure of hemostasis in the operation after the autopsy. One rat in the SIS group presented with a large ventral hernia and severe nutritional marasmus on the $7^{\text {th }}$ day. The rat was euthanized and autopsy indicated that the sutures unclamped, resulting in bowel herniation and obstruction. Bulge was observed in 2 animals in the SPS group and in 4 animals in the SIS group between 7 and 14 days. No bulge or herniation appeared in the SVDPS and SVPS groups.

To evaluate the quality of the repair, animals were euthanized and the repair areas were visually observed in each group. The adhesion level weakened gradually with time and the adhesion surface and tenacity between groups showed no significant difference throughout the entire experimental period (Fig. 4E and F, $p>0.05$ ). The average thickness of the explants in the SVDPS group was close to that of the SVPS and SPS groups without significant difference, while all of them were significantly thicker than that of the SIS group after 1 month post-surgery (ESI Fig. $1, \dagger p<0.05$ ). At 12 weeks after implantation, the surface area of the SVDPS and SVPS showed contraction by $25 \pm 4 \%$ and $27 \pm 3 \%$. Furthermore, the surface area of SPS and SIS showed contraction by $29 \pm 5 \%$ and $25 \pm 3 \%$ compared with the areas of the original implants 

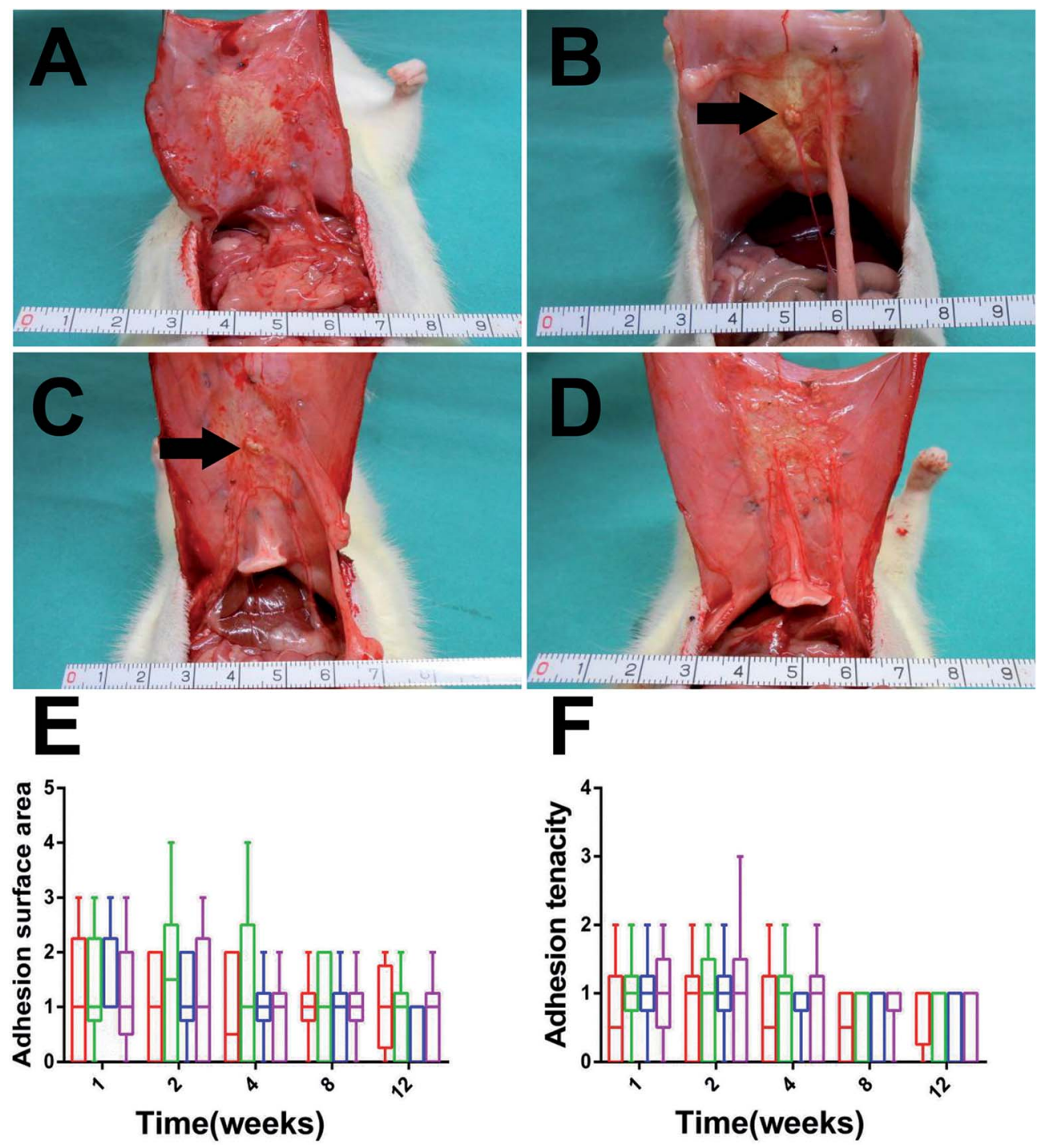

Fig. 4 Characteristics of the implanted scaffolds. Macroscopy of the explants of SIS (A), SPS (B), SVPS (C), and SVDPS (D) at 3 months postoperation. Black arrow indicates the remaining PLGA. (E and F) The adhesion area and tenacity showed no significant difference among the groups.

$\left(12 \mathrm{~cm}^{2}, p<0.01\right)$. Whileras, there were no significant variation observed in among all the groups by 12 weeks after implantation (ESI Fig. $2, \dagger p>0.05$ ).

3.7.2 Histological and immunohistochemical examination. To further assess the repair efficiency at the histological and immunohistochemical levels, the collagen deposition, the density of blood vessels and the inflammatory response were assessed. Masson trichrome staining showed that SVDPS group induced more intense collagen deposition from 2 to 12 weeks than the other 3 groups (Fig. 5). In addition, both SVDPS and SVPS explants showed a greater collagen deposition at the 4 week point than SIS and SPS groups (SVDPS, $p<0.05$; SVPS, $p<$ 0.01). At the last time point, 12 weeks after implantation, there were no differences exhibited among the SIS, SPS and SVPS (ESI Fig. $3, \dagger p>0.05$ ).

CD31 staining showed the density of capillary in the SVDPS and SVPS groups were significantly higher than that in the SIS group in the first week post-surgery $\left(162.06 \pm 30.12 / \mathrm{mm}^{2}\right.$, $133.28 \pm 31.42 / \mathrm{mm}^{2}, 95.32 \pm 40.01 / \mathrm{mm}^{2}$, and $90.49 \pm 37.08 /$ $\mathrm{mm}^{2}$, respectively; $\left.p<0.0001\right)$. Moreover, the level of SVDPS group was higher than that of SPS group $(p<0.001)$. In the second week, the capillary density in the SVDPS group was remarkably higher than all the other groups $(p<0.001)$ (Fig. 6I). However, the vascular density in the SVPS group decreased to a level similar to that of the SPS and SIS groups. From the fourth week after surgery, a similar density of vessels was observed among all groups without statistical difference (Fig. 6A-H).

As shown by western blotting in Fig. 6J, the SVDPS group showed prominent CD31 expression compared to the other groups $(p<0.01)$, indicating higher levels of early vascularization. Although VEGF was loaded into SVPS, no significant difference was observed when compared with the SPS and SIS groups, and this may be due to the low encapsulation efficiency and VEGF bioactivity in the SVPS group. 


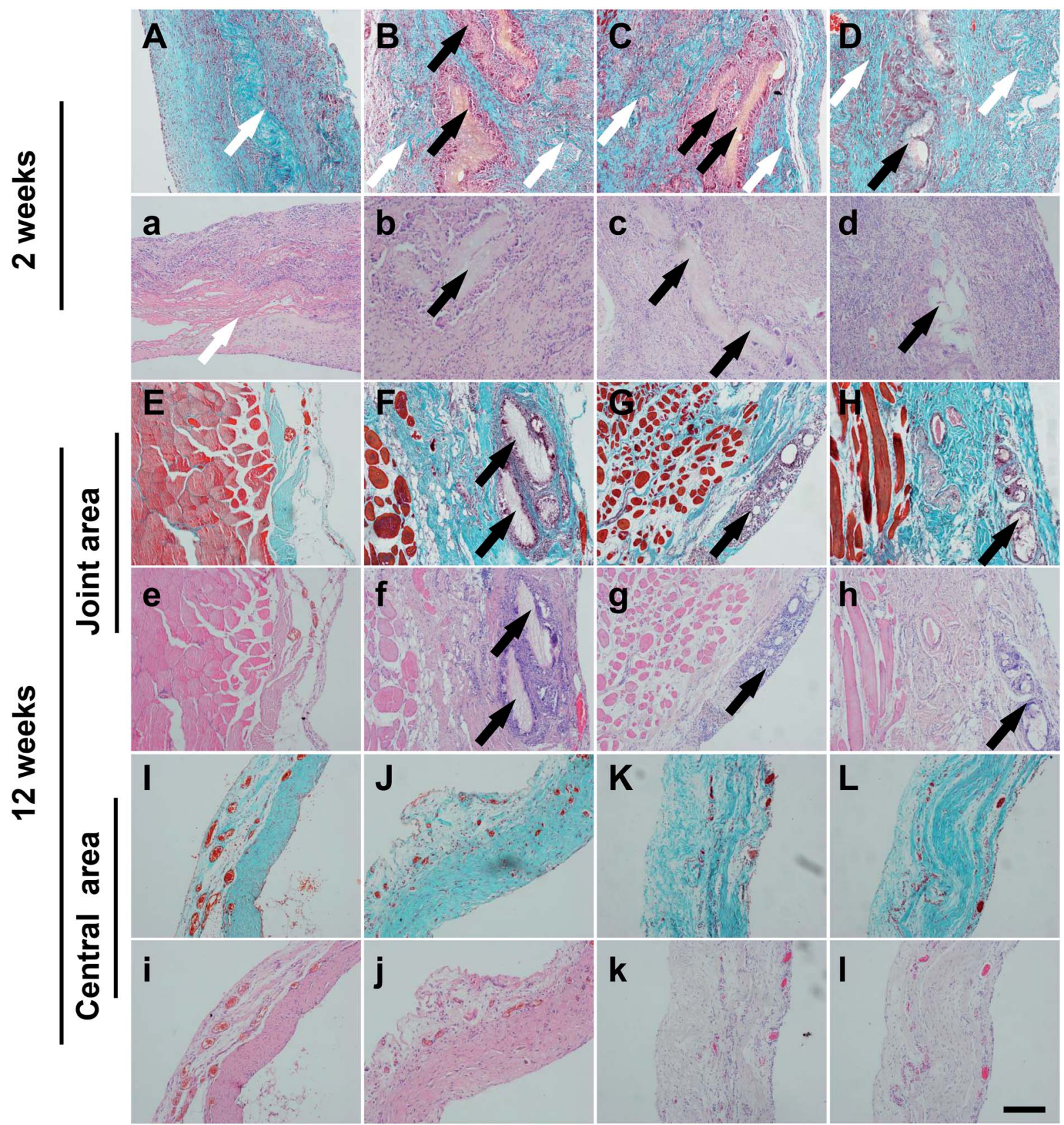

Fig. $5 \mathrm{H} \& \mathrm{E}(\mathrm{a}-\mathrm{l})$ and Masson's trichrome staining (A-L) of different explants post-implantation. At 2 weeks, slow PLGA and SIS degradation, less collagen deposition, and fewer newly formed blood vessels were observed at the interface between PLGA and SIS in the SPS (B,b) and SVPS (C,C) groups, while rapid PLGA and SIS degradation, pronounced new collagen fibers, and newly formed blood vessels were observed at the interface between PLGA and SIS in the SVDPS group (D,d). At 3 months, oriented bundles of collagen fibers with abundant blood vessels were observed both at the joint and the central area in all groups $(E-L, e-l)$, while less PLGA residues and inflammatory response was observed in the SVDPS group (H,h: joint area; L,l: central area) when compared with the SVPS (G, g: joint area; K,k: central area) or SPS group (F,f: joint area; J,j: central area). SIS (A,a; E,e; l,i) was used as a control. The black arrows and the white arrows indicate PLGA and SIS, respectively. Magnification: $100 \times$, scale bar $=200 \mu \mathrm{m}$.

FSP1 staining (Fig. 7) revealed that the maximum number of fibroblasts was observed during the first week post-surgery in all groups without statistical difference. At 2 weeks post-surgery, more fibroblasts were present at the repair site in the SVDPS group when compared to the other groups $(1349.65 \pm 334.37 /$ $\mathrm{mm}^{2}, 898.64 \pm 229.32 / \mathrm{mm}^{2}, 975.95 \pm 166.55 / \mathrm{mm}^{2}$, and 1008.45 $\pm 311.45 / \mathrm{mm}^{2}$, respectively; $p<0.0001$ ). Intriguingly, Masson's trichrome staining (Fig. 5a-l) revealed higher collagen deposition and less PLGA and SIS remnants in the SVDPS group when compared with those in the SVPS and SPS groups from 2 to 4 weeks post-surgery, though the thickness of SVDPS explants was similar to that of SVPS and SPS explants. 

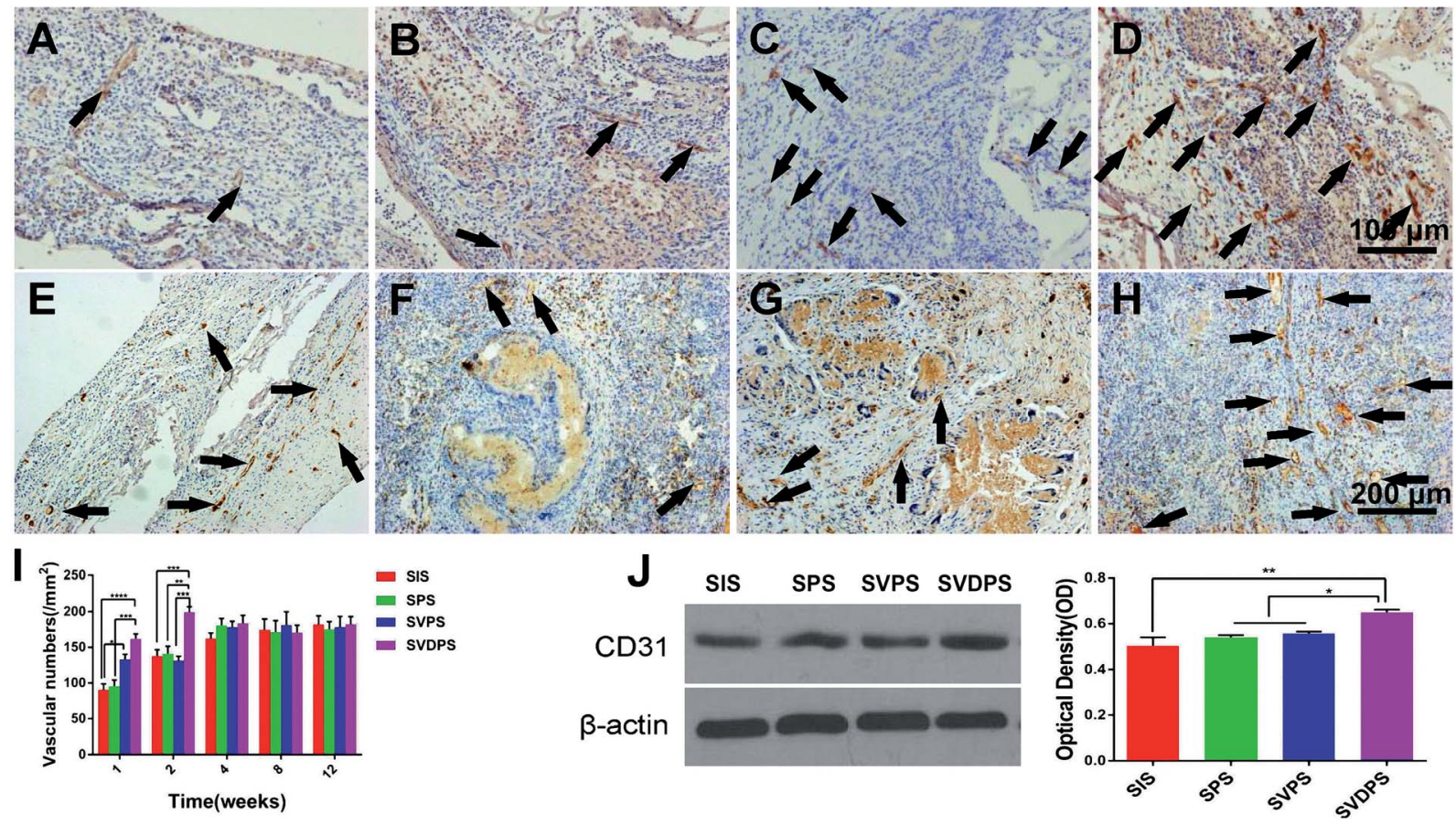

Fig. 6 Immunohistochemical staining for CD31 of SIS, SPS, SVPS, and SVDPS explants at 1 week (A-D) and 2 weeks (E-H) post-surgery. A greater density of blood vessels was observed in the SVDPS group at 1 week (D) and 2 weeks (H) when compared with the SIS (A, E), SPS (B, F), and SVPS groups (C, G). The black arrows indicate positive CD31. (I) CD31 positive vascular density at 12 weeks. (J) Western blot analysis confirmed that CD31 was higher in the SVDPS group. Magnification: (A-D) $200 \times$, scale bar $=100 \mu \mathrm{m} ;(E-H) 100 \times$. Scale bar $=200 \mu \mathrm{m}$. ****P<0.0001; ***P< $0.001 ; * * P<0.01 ; * P<0.05$.
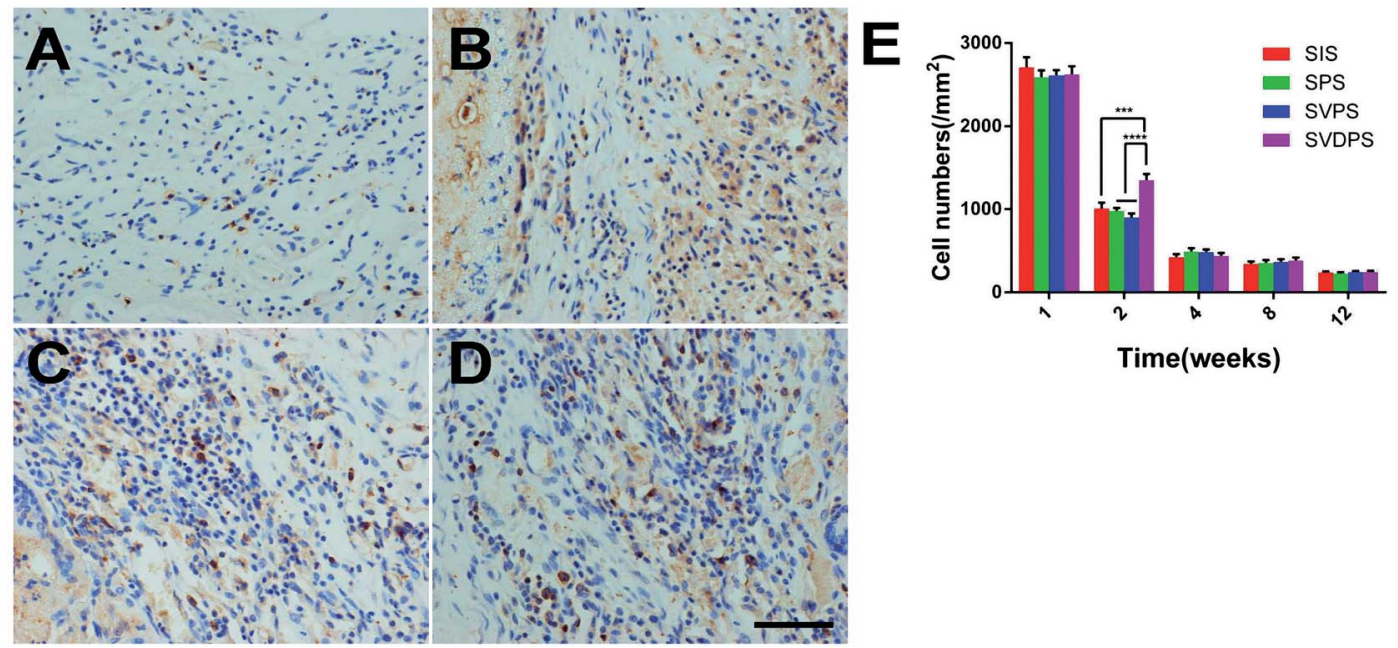

Fig. 7 Immunohistochemical staining for FSP-1 of SIS (A), SPS (B), SVPS (C), and SVDPS (D) explants at 2 weeks post-surgery. A greater density of fibroblasts was observed in the SVDPS group compared with that in the SIS, SPS, and SVPS groups. (E) Quantification of fibroblasts at 12 weeks. Magnification: $400 \times$. Scale bar $=50 \mu \mathrm{m}$. ****P<0.0001; ***P<0.001.

Macrophages were stained (Fig. 8) to assess the inflammation induced by implants. PLGA-involved materials induced more severe inflammatory response than SIS in 8 weeks $(p<0.001)$, while no significant difference was observed among the SVDPS, SVPS, and SPS groups.

3.7.3 Mechanical property analysis. In this study, the maximal load, Young's modulus, tensile strength and stiffness serve as the parameters for mechanical property analysis. In the first week post-surgery, statistical analysis showed no significant difference in the max load, Young's modulus, tensile strength and stiffness among four groups (Fig. 9, ESI Table $1, \dagger$ $p>0.05$ ). For in vivo mechanical properties, the max load, tensile strength and stiffness of SVDPS, SVPS, SPS and SIS decreased significantly in the first week $(p<0.05)$. Although 

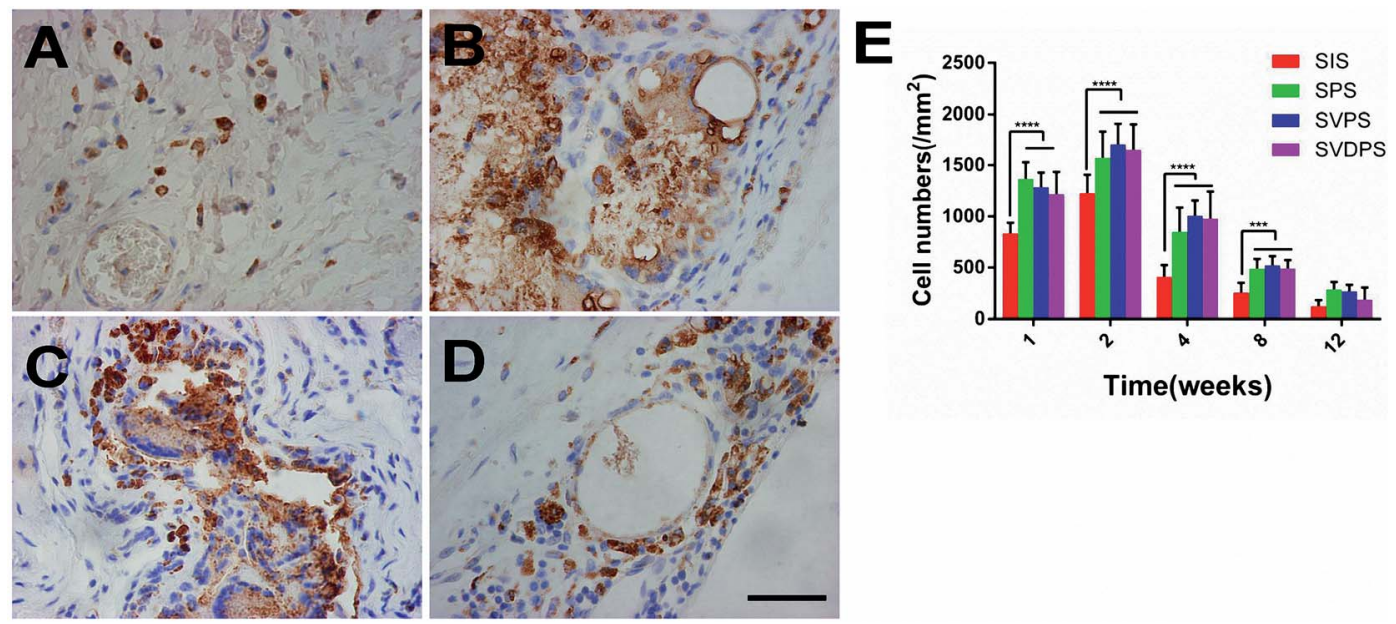

Fig. 8 Immunohistochemical staining for CD68 of SIS (A), SPS (B), SVPS (C), and SVDPS (D) explants at 12 weeks post-surgery. (E) Quantification of CD68 positive macrophages at 12 weeks. The scaffolds in the PLGA-involved groups induced more severe inflammatory response than those from the SIS group at 8 weeks, while it was reduced at 12 weeks. Magnification: $400 \times$. Scale bar $=50 \mu \mathrm{m}$. ****P<0.0001;***P<0.001.

A

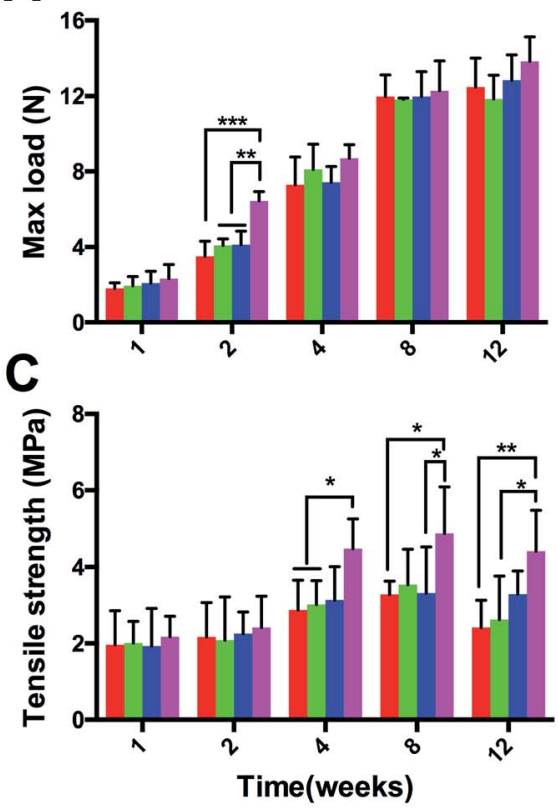

B
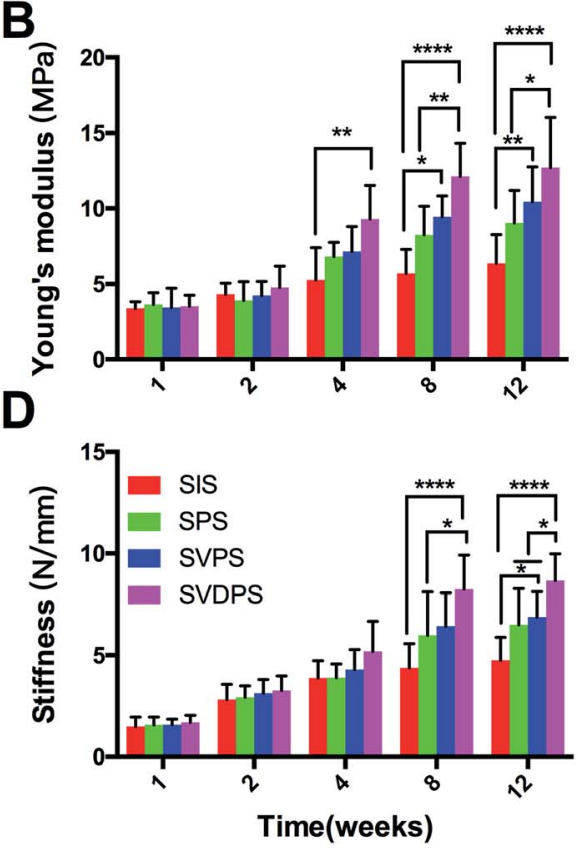

Fig. 9 Mechanical property analysis of the explants. Quantitative analyses of max load (A), Young's modulus (B), tensile strength (C) and stiffness (D) among SVDPS, SVPS, SPS and SIS groups at 1, 2, 4, 8 and 12 weeks post-implantation time points. $* P<0.05, * * P<0.01, * * * * P<0.0001$.

Young's modulus decreased, it showed no statically significance between the different groups. However, the reported values do not actually represent the strength of the explants, but rather represent the degree of integration at the interface with the surrounding tissues, because all explants failed at the joint area at 1 week after implantation.

After 1 week post-surgery, the mechanical properties of all scaffolds started to gradually increase (Fig. 9, ESI Tables 1-5†), consistent with the collagen fiber deposition. From the second week, almost all samples were broken in the central area, which indicated that the tissue incorporation had improved. As shown in Fig. 9A, the max load at 2 weeks post-implantation for SVDPS explants was statistically higher than that of SVPS, SPS, and SIS explants. Thereafter, from 4 to 12 weeks post-surgery, no significant difference was observed among four groups as revealed by statistical analysis $(p>0.05)$.

As revealed in Fig. 9B and ESI Tables $1-5, \dagger$ the Young's modulus at 2 weeks post-surgery for SVDPS, SVPS, SPS, and SIS groups showed no significant difference $(p>0.05)$. At 4 weeks post-surgery, the Young's modulus of SVDPS group was significantly higher than that of SIS group $(p<0.001)$. Moreover, at both time points of 8 and 12 weeks post-surgery, the Young's 
modulus of SVDPS group significantly higher than that of SPS and SIS groups $(p<0.001)$. In addition, the value of SVPS group was also significantly higher than that of SIS group $(p<0.05)$.

As revealed in Fig. 9C and ESI Tables $1-5, \dagger$ the tensile strength at 2 weeks post-surgery for SVDPS, SVPS, SPS, and SIS groups showed no significant difference among four groups $(p>$ 0.05). At 4 weeks post-surgery, the tensile strength of SVDPS group was significantly higher than that of SPS and SIS groups $(p<0.05)$. And at 8 weeks post-surgery, the tensile strength of SVDPS group was significantly higher than that of SVPS and SIS groups $(p<0.05)$. At 12 weeks post-surgery, SVDPS group was significantly higher than that of SPS and SIS groups $(p<0.05)$.

As revealed in Fig. 9D, the stiffness at 2 and 4 weeks postsurgery for SVDPS, SVPS, SPS, and SIS groups showed no significant difference among four groups $(p>0.05)$. At 8 weeks post-surgery, the stiffness for SVDPS, SVPS, SPS, and SIS groups were respectively $8.25 \pm 1.67 \mathrm{~N} \mathrm{~mm}^{-1}, 6.43 \pm 1.64 \mathrm{~N} \mathrm{~mm}^{-1}, 5.99$ $\pm 2.14 \mathrm{~N} \mathrm{~mm}^{-1}$ and $4.38 \pm 1.18 \mathrm{~N} \mathrm{~mm}^{-1}$ (ESI Table $4 \dagger$ ) and the stiffness of SVDPS group was significantly higher than that of SPS and SIS groups $(p<0.05)$. At 12 weeks post-surgery, the stiffness for SVDPS was significantly higher than that of SVPS, SPS, and SIS groups, which were respectively $8.67 \pm 1.32 \mathrm{~N}$ $\mathrm{mm}^{-1}, 6.88 \pm 1.25 \mathrm{~N} \mathrm{~mm}^{-1}, 6.49 \pm 1.78 \mathrm{~N} \mathrm{~mm}^{-1}$ and $4.76 \pm$ $1.12 \mathrm{~N} \mathrm{~mm}^{-1}$ (ESI Table $5, \dagger p<0.05$ ). In addition, the stiffness of SVPS group was also significantly higher than that of SIS group $(p<0.05)$.

\section{Discussion}

Previous studies reported that neovascularization could be induced by either transplantation of mesenchymal stem cell $^{33,34}$ or sustained delivery of angiogenic factors. ${ }^{35,36}$ With regard to large abdominal wall defects, it is more emergent and complicated for the isolation, proliferation, and seeding of autologous stem cells. Hence, we considered that the introduction of exogenous pro-angiogenic GFs is a more feasible measure. So a novel controlled release system consisting of PLGA (as the GF carrier), dextran (as the GF protective agent), and VEGF is developed. In this system, VEGF was encapsulated and protected by DNPs without any contact with the organic solvent and polymer solution or exposure to high temperatures. Compared to the emulsion method, VEGF bioactivity was retained even after the formulated VEGF/DNPs were further electrospun into the PLGA membrane, as demonstrated by the stimulation HUVEC proliferation in vitro. Furthermore, the VEGF/DNPs-PLGA membrane in which VEGF was protected by dextran presented no severe burst release and a controlled release kinetic of nearly 20 days. However, VEGF was released from VEGF/PLGA membranes within a short duration of approximately 14 days. These findings confirmed that our system could effectively protect VEGF bioactivity and allowed VEGF controlled release.

VEGF is a mitogen to ECs and acts specifically and potently on ECs to induce their migration, proliferation, and the formation of new vessels. ${ }^{37}$ We further determined the in vivo angiogenic efficiency of the exogenous VEGF released from VEGF/DNPs-PLGA and VEGF-PLGA and verified the role of early vascularization. In the first 2 weeks post-implantation, the SVDPS membrane showed a significant potential in promoting angiogenesis when compared with the other three groups. However, only an intermediate level of new microvessels at 1 week was observed in the SVPS group in which VEGF was unprotected. The physiological growth rate of newly developing neovessels is slow and it is estimated to be no faster than $5 \mu \mathrm{m}$ $\mathrm{h}^{-1} \cdot{ }^{38}$ Sufficient blood supply cannot be completely achieved by the natural ingrowth of blood vessels from the surrounding tissues. ${ }^{39}$ In the present study, early neovascularization was successfully established by controlled release of exogenous VEGF in the SVDPS group within 2 weeks, indicating that VEGF bioactivity was efficiently preserved by our method.

The formation and growth of new tissues are decisively dependent on the rapid establishment of a sufficient blood supply, which provides with a continuous blood flow for the delivery of oxygen and nutrients and for the elimination of waste products. ${ }^{40}$ In the present study, Masson's trichrome revealed an interesting difference among the groups. The SVDPS group presented significantly higher collagen content than the SVPS, SPS and SIS groups from 2 to 12 weeks. Futhermore, SVPS group also showed a greater collagen deposition at the 4 week point than SIS and SPS groups. It indicated that VEGF releasing actually improved the collagen deposition, and the effort was enhanced more intensively owing to the DNPs in SVDPS group. New collagen are matured and remodeled from pre-collagen that is initially secreted by fibroblasts. Thus, we assessed fibroblast infiltration by immunohistochemistry and confirmed that the number of fibroblasts, was significantly higher in the SVDPS group within 2 weeks, while no significant difference was observed among all groups at other time points. Because VEGF cannot directly induce fibroblasts proliferation, we consider that their propagation was indirectly promoted by increased blood supply. High level of vascularization not only promoted new tissue regeneration, but also accelerated the degradation of the implants. ${ }^{13}$ Early angiogenesis in the SVDPS group led to increased infiltration of host cells and, consequently, quicker SIS degradation when compared to the other groups. Our results proved that sufficient early neovascularization provoked by VEGF release from SVDPS significantly enhanced the speed of new tissue ingrowth as well as implant degradation.

Successful repair of abdominal wall defect depends on sufficient mechanical properties to prevent postoperative herniation or bulge. If the processes of new tissue regeneration and remodeling outpace mesh degradation, the herniation or bulge rate would be significantly reduced. The difference in new tissue regeneration and remodeling was reflected by the difference in mechanical properties of examined tissue samples. In this study, the mechanical examination at 1 week after operation showed that all explants failed at the junction of the scaffolds and the surrounding tissues, indicating that new tissue regeneration and remodeling is insufficient, thus the mechanical properties of all samples were weak at this time point. At the second week post-surgery, the max load of SVDPS at 2 weeks post-surgery was significantly higher than that of the other groups. The Young's modulus, tensile strength and 
stiffness were also stronger than those of the other groups in the irregular periods, although the difference was not statistically significant at some time point. These mechanical properties indicates that new tissue regeneration and remodeling were significantly enhanced in SVDPS group since 2 weeks postsurgery, which was consistent with collagen deposition. These mechanical properties were also reflected by the amount of bulges post-surgery. Bulges was observed in 2 and 4 animals in the SPS and SIS groups between 7 and 14 days post-surgery, respectively, while no herniation or bulge was observed in the SVDPS and SVPS groups. This was mostly because the early therapeutic angiogenesis induced fast new collagen deposition and tissue remodeling, which transcended the degradation of materials. According to this result, we believe that early neovascularization in the first 2 weeks post-surgery is crucial and indispensable for successful abdominal wall defect repair.

A previous study indicated that PLGA induced obvious postsurgery inflammation when compared to SIS. ${ }^{6}$ Inflammation may cause visceral adhesion, seroma formation, and may even play a role in new vessel formation. In our study, levels of inflammation represented by macrophage aggregation was enhanced in SVDPS, SVPS, and SPS groups when compared to the SIS group at 2 weeks and gradually decreased with time, while no significant difference was observed among these PLGA-involved groups. Both adhesion and seroma formation showed no statistical difference among groups, indicating that the inflammation provoked by PLGA was acceptable. Moreover, similar levels of inflammation demonstrated that inflammation does not significantly influence angiogenesis when compared with the introduction of exogenous VEGF.

\section{Conclusion}

In summary, we exploited a carrier to obtain a sustained release of bioactive VEGF to improve early neovascularization of SIS repaired abdominal wall. In vitro, VEGF showed sustained release properties and better bioactivity and stability under the protection of dextran. In vivo, early neovascularization were promoted by the VEGF released from the composite scaffold and consequently, the collagen deposition, tissue remodeling were improved accordingly. The mechanical properties in variable quantities were also improved in different periods. This system may be considered to modify the original SIS to a bioactive material and such types of modifications may improve the clinical efficacy of abdominal wall defect repair in the near future.

\section{Author contribution statement}

Rui Tang, Xin Wang wrote the main manuscript text. Hanying Zhang, Xi Liang prepared the in vitro study. Xueyi Feng, Xiaoqiang Zhu prepared the in vivo study. Xinwu Lu, Fei Wu, Zhengni Liu modified the paper and checked the data.

\section{Conflicts of interest}

The authors report no conflicts of interest in this work.

\section{Acknowledgements}

This work was supported by the Medical Guidance Project of Shanghai Science and Technology Commission (grant number 15411962500).

\section{References}

1 G. E. Leber, J. L. Garb, A. I. Alexander and W. P. Reed, Longterm complications associated with prosthetic repair of incisional hernias, Arch. Surg., 1998, 133(4), 378-382.

2 L. Ansaloni, F. Catena, F. Coccolini, F. Gazzotti, L. D'Alessandro, et al., Inguinal hernia repair with porcine small intestine submucosa: 3-year follow-up results of a randomized controlled trial of Lichtenstein's repair with polypropylene mesh versus Surgisis Inguinal Hernia Matrix, Am. J. Surg., 2009, 198(3), 303-312.

3 K. Junge, M. Binnebösel, R. Rosch, M. Jansen, D. Kämmer, et al., Adhesion formation of a polyvinylidenfluoride/ polypropylene mesh for intra-abdominal placement in a rodent animal model, Surg. Endosc., 2009, 23(2), 327-333.

4 J. Meintjes, S. Yan, L. Zhou, S. Zheng and M. Zheng, Synthetic, biological and composite scaffolds for abdominal wall reconstruction, Expert Rev. Med. Devices, 2011, 8(2), 275-288.

5 C. Birolini, J. G. Minossi, C. F. Lima, E. M. Utiyama and S. Rasslan, Mesh cancer: long-term mesh infection leading to squamous-cell carcinoma of the abdominal wall, Hernia, 2014, 18(6), 897-901.

6 M. S. Kim, H. H. Ahn, Y. N. Shin, M. H. Cho, G. Khang, et al., An in vivo study of the host tissue response to subcutaneous implantation of PLGA- and/or porcine small intestinal submucosa-based scaffolds, Biomaterials, 2007, 28(34), 5137-5143.

7 S. Wu, Y. Liu, S. Bharadwaj, A. Atala and Y. Zhang, Human urine-derived stem cells seeded in a modified 3D porous small intestinal submucosa scaffold for urethral tissue engineering, Biomaterials, 2011, 32(5), 1317-1326.

8 F. Zafar, R. B. Hinton, R. A. Moore, R. S. Baker, R. Bryant, et al., Physiological Growth, Remodeling Potential, and Preserved Function of a Novel Bioprosthetic Tricuspid Valve: Tubular Bioprosthesis Made of Small Intestinal Submucosa-Derived Extracellular Matrix, J. Am. Coll. Cardiol., 2015, 66(8), 877-888.

9 D. Bryant, R. Holtby, K. Willits, R. Litchfield, D. Drosdowech, et al., A randomized clinical trial to compare the effectiveness of rotator cuff repair with or without augmentation using porcine small intestine submucosa for patients with moderate to large rotator cuff tears: a pilot study, J. Shoulder Elbow Surg., 2016, 25(10), 1623-1633.

10 J. D. Vaught, B. P. Kropp, B. D. Sawyer, M. K. Rippy, S. F. Badylak, et al., Detrusor regeneration in the rat using porcine small intestinal submucosal grafts: functional innervation and receptor expression, J. Urol., 1996, 155(1), 374-378.

11 Z. Liu, X. Feng, H. Wang, J. Ma, W. Liu, et al., Carbon nanotubes as VEGF carriers to improve the early 
vascularization of porcine small intestinal submucosa in abdominal wall defect repair, Int. J. Nanomed., 2014, 10(9), 1275-1286.

12 N. J. Smart and S. Bloor, Durability of biologic implants for use in hernia repair: a review, Surg. Innovat., 2012, 19(3), 221-229.

13 Z. Song, Z. Yang, J. Yang, Z. Liu, Z. Peng, et al., Repair of abdominal wall defects in vitro and in vivo using VEGF sustained-release multi-walled carbon nanotubes (MWNT) composite scaffolds, PLoS One, 2013, 8(5), e64358.

14 Z. Liu, R. Tang, Z. Zhou, Z. Song, H. Wang, et al., Comparison of two porcine-derived materials for repairing abdominal wall defects in rats, PLoS One, 2011, 6(5), e20520.

15 J. Zhang, G. Y. Wang, Y. P. Xiao, L. Y. Fan and Q. Wang, The biomechanical behavior and host response to porcinederived small intestine submucosa, pericardium and dermal matrix acellular grafts in a rat abdominal defect model, Biomaterials, 2011, 32(29), 7086-7095.

16 M. Ditzel, E. B. Deerenberg, N. Grotenhuis, J. J. Harlaar, K. Monkhorst, et al., Biologic meshes are not superior to synthetic meshes in ventral hernia repair: an experimental study with long-term follow-up evaluation, Surg. Endosc., 2013, 27(10), 3654-3662.

17 C. H. Ho, P. W. Liao, S. S. Yang, F. S. Jaw and Y. C. Tsai, The use of porcine small intestine submucosa implants might be associated with a high recurrence rate following laparoscopic herniorrhaphy, J. Formosan Med. Assoc., 2015, 114(3), 216-220.

18 M. Miserez, G. Grass, C. Weiss, H. Stützer, S. Sauerland, et al., Closure of the LAPSIS trial, Br. J. Surg., 2010, 97(10), 1598.

19 T. Simon-Yarza, E. Tamayo, C. Benavides, H. Lana, F. R. Formiga, et al., Functional benefits of PLGA particulates carrying VEGF and CoQ10 in an animal of myocardial ischemia, Int. J. Pharm., 2013, 454(2), 784-790.

20 B. Akar, B. Jiang, S. I. Somo, A. A. Appel, J. C. Larson, et al., Biomaterials with persistent growth factor gradients in vivo accelerate vascularized tissue formation, Biomaterials, 2015, 72, 61-73.

21 J. S. Park, H. N. Yang, S. W. Yi, J. H. Kim and K. H. Park, Neoangiogenesis of human mesenchymal stem cells transfected with peptide-loaded and gene-coated PLGA nanoparticles, Biomaterials, 2016, 76, 226-237.

22 T. Morita, Y. Horikiri, H. Yamahara, T. Suzuki and H. Yoshino, Formation and isolation of spherical fine protein microparticles through lyophilization of proteinpoly(ethylene glycol) aqueous mixture, Pharm. Res., 2000, 17(11), 1367-1373.

23 U. Bilati, E. Allemann and E. Doelker, Strategic approaches for overcoming peptide and protein instability within biodegradable nano- and microparticles, Eur. J. Pharm. Biopharm., 2005, 59(3), 375-388.

24 L. Jorgensen, E. H. Moeller, M. van de Weert, H. M. Nielsen and S. Frokjaer, Preparing and evaluating delivery systems for proteins, Eur. J. Pharm. Sci., 2006, 29(3-4), 174-182.

25 F. Wu, Z. Zhou, J. Su, L. Wei, W. Yuan, et al., Development of dextran nanoparticles for stabilizing delicate proteins, Nanoscale Res. Lett., 2013, 8(1), 197.
26 T. Jin, H. Zhu and J. Zhu, Hazard-free microencapsulation for structurally delicate agents, an application of stable aqueous-aqueous emulsion, EP, CA 2487867 A1, 2003.

27 S. Liu, M. Qin, C. Hu, F. Wu, W. Cui, et al., Tendon healing and anti-adhesion properties of electrospun fibrous membranes containing bFGF loaded nanoparticles, Biomaterials, 2013, 34(19), 4690-4701.

28 W. Yuan, F. Wu, Y. Geng, S. Xu and T. Jin, Preparation of dextran glassy particles through freezing-induced phase separation, Int. J. Pharm., 2007, 339(1-2), 76-83.

29 L. Luo, Y. He, Q. Chang, G. Xie, W. Zhan, et al., Polycaprolactone nanofibrous mesh reduces foreign body reaction and induces adipose flap expansion in tissue engineering chamber, Int. J. Nanomed., 2016, 11, 6471-6483.

30 Y. Liu, B. Ml, J. Wiltfang, P. H. Warnke and H. Terheyden, Tissue engineering of a vascularized bone graft of critical size with an osteogenic and angiogenic factor-based in vivo bioreactor, Tissue Eng., Part A, 2014, 20(23-24), 3189-3197.

31 F. Strutz, H. Okada, C. W. Lo, T. Danoff, R. L. Carone, et al., Identification and characterization of a fibroblast marker: FSP1, J. Cell Biol., 1995, 130(2), 393-405.

32 W. Wang, J. He, B. Feng, Z. Zhang, W. Zhang, et al., Aligned nanofibers direct human dermal fibroblasts to tenogenic phenotype in vitro and enhance tendon regeneration in vivo, Nanomedicine, 2016, 11(9), 1055-1072.

33 B. Wang, W. Liu, Y. Zhang, Y. Jiang, W. J. Zhang, et al., Engineering of extensor tendon complex by an ex vivo approach, Biomaterials, 2008, 29(20), 2954-2961.

34 T. S. Iyyanki, L. W. Dunne, Q. Zhang, J. Hubenak, K. C. Turza, et al., Adipose-derived stem-cell-seeded noncross-linked porcine acellular dermal matrix increases cellular infiltration, vascular infiltration, and mechanical strength of ventral hernia repairs, Tissue Eng., Part A, 2015, 21(3-4), 475-485.

35 S. Danner, M. Kremer, A. E. Petschnik, S. Nagel, Z. Zhang, et al., The use of human sweat gland-derived stem cells for enhancing vascularization during dermal regeneration, $J$. Invest. Dermatol., 2012, 132(6), 1707-1716.

36 J. H. Kim, Y. Jung, B. S. Kim and S. H. Kim, Stem cell recruitment and angiogenesis of neuropeptide substance $\mathrm{P}$ coupled with self-assembling peptide nanofiber in a mouse hind limb ischemia model, Biomaterials, 2013, 34(6), 1657-1668.

37 S. S. Said, J. G. Pickering and K. Mequanint, Advances in growth factor delivery for therapeutic angiogenesis, J. Vasc. Res., 2013, 50(1), 35-51.

$38 \mathrm{H}$. A. Zarem, The microcirculatory events within fullthickness skin allografts (homografts) in mice, Surgery, 1969, 66(2), 392-397.

39 A. W. Orr, C. A. Elzie, D. F. Kucik and J. E. Murphy-Ullrich, Thrombospondin signaling through the calreticulin/LDL receptor-related protein co-complex stimulates random and directed cell migration, J. Cell Sci., 2003, 116(Pt14), 2917-2927.

40 M. W. Laschke, C. Grässer, S. Kleer, C. Scheuer, D. Eglin, et al., Adipose tissue-derived microvascular fragments from aged donors exhibit an impaired vascularisation capacity, Eur. Cells Mater., 2014, 28, 287-298. 